\title{
Codimension-three bifurcations in a Bénard-Marangoni problem
}

\author{
Sergio Hoyas, ${ }^{1, *}$ Antonio Gil, ${ }^{1}$ Pablo Fajardo, ${ }^{1,2}$ and María J. Pérez-Quiles ${ }^{3}$ \\ ${ }^{1}$ CMT-Motores Térmicos, Universitat Politècnica de València, 46022 València, Spain \\ ${ }^{2}$ Bioingeniería e Ingeniería Aeroespacial, Universidad Carlos III de Madrid, 28911 Leganés, Spain \\ ${ }^{3}$ Instituto Universitario de Matemática Pura y Aplicada, Universitat Politècnica de València, 46022 València, Spain
}

(Received 15 March 2013; revised manuscript received 12 June 2013; published 22 July 2013)

\begin{abstract}
This Brief Report studies the linear stability of a thermoconvective problem in an annular domain for relatively low $(\sim 1)$ Prandtl (viscosity effects) and Biot (heat transfer) numbers. The four possible patterns for the instabilities, namely, hydrothermal waves of first and second class, longitudinal rolls, and corotating rolls, are present in a small region of the Biot-Prandtl plane. This region can be split in four zones, depending on the sort of instability found. The boundary of these four zones is composed of codimension-two points. Authors have also found two codimension-three points, where some of the former curves intersect. Results shown in this Brief Report clarify some reported experiments, predict new instabilities, and, by giving a deeper insight into how physical parameters affect bifurcations, open a gateway to control those instabilities.
\end{abstract}

DOI: 10.1103/PhysRevE.88.015001

PACS number(s): 47.20.Bp, 47.20.Ky, 47.35.Bb

The problem of thermoconvective instabilities in fluid layers driven by a temperature gradient has become a classical subject in fluid mechanics [1,2]. In the case of BénardMarangoni convection problems, the effects of both gravity and capillary forces are taken into account. Classically, heat is applied from below [1]. A more general setup may be considered which includes thermoconvective instabilities by imposing a basic dynamic flow through temperature gradients with a horizontal component, either in rectangular geometries [2-4] or in cylindrical and annular geometries [5-9].

In this sort of problem there are many nondimensional numbers controlling the shape and evolution of the instabilities. Apart from the geometrical parameters, the classical numbers, which are described below, are Rayleigh (thermal effects), Marangoni (surface tension), Bond (their ratio), Prandtl (viscous to thermal diffusivity ratio), and Biot (heat exchange) numbers. Experimentally, for the same fluid, Rayleigh and Marangoni are easy to change, just increasing the heat present in the system. In contrast, Prandtl and Biot numbers are more difficult to control and, as it is shown below, a great richness of bifurcations appears depending only on them.

The physical domain is presented in Fig. 1. A horizontal fluid layer of depth $d$ (the $z$ coordinate) is contained in the space limited by two concentric cylinders of radii $a$ and $a+\delta$ (the $r$ coordinate). For the numerical experiments shown here, the aspect ratio $\Gamma=\delta / d$ is set to 4 and $a=\delta$. The lateral walls are both adiabatic, and the top is open to the atmosphere. A decreasing linear temperature profile from the inner to the outer cylinder was imposed on the bottom plate. The horizontal temperature gradient at the bottom had a value of $T_{G}=2.2 \mathrm{~K}$ and remained constant throughout the computations.

The system evolves according to the momentum and mass balance equations and to the energy conservation principle, nondimensionalized as in $[5,10]$. As usual in this sort of problem, the Boussinesq approximation was used [10]. In the equations governing the system, $u_{r}, u_{\theta}$, and $u_{z}$ are the components

\footnotetext{
*serhocal@ mot.upv.es
}

of the velocity field $\mathbf{u}, \Theta$ is the temperature, and $p$ is the pressure. The nondimensionalized equations then become

$$
\begin{gathered}
\nabla \cdot \mathbf{u}=0 \\
\partial_{t} \mathbf{u}+(u \cdot \nabla) \mathbf{u}=\operatorname{Pr}\left(\nabla p+\nabla^{2} \mathbf{u}+\operatorname{Ra} \Theta \mathbf{e}_{z}\right), \\
\partial_{t} \Theta+\mathbf{u} \cdot \nabla \Theta=\nabla^{2} \Theta .
\end{gathered}
$$

In these equations the operators and fields are expressed in cylindrical coordinates and $\mathbf{e}_{z}$ is the unit vector in the $z$ direction.

Boundary conditions (BCs) are similar to those of $[5,11]$. They are summarized in Table I. Briefly, the velocity is zero on the cylinders and the bottom plate. In the top surface, the thermocapillarity forces are modeled through the Marangoni condition [5]. The Biot condition has been used to model the heat transfer to the atmosphere.

In Eqs. (1)-(3) and BCs given by Table I the nondimensional numbers introduced previously are used. These numbers depend on the thermal diffusivity $\kappa$, the kinematic viscosity $v$, the thermal expansion coefficient $\alpha$, the heat transfer coefficient $h$, and the gravitational acceleration $g$. The Rayleigh number, $\mathrm{Ra}=g \alpha \Delta T d^{4} / \kappa \nu$, is representative of the buoyancy effect, and it is the control parameter used in this work. The Marangoni number, which accounts for the surface tension effects, is defined as Ma $=\gamma \Delta T d^{2} / \rho \kappa \nu$. The ratio between these two numbers, the Bond number Bo, is kept constant, so that Bo $\sim 70$ and therefore buoyancy effects are dominant. Two more numbers play an important role. First, the Prandtl number, $\operatorname{Pr}=v / \kappa$, is the ratio of momentum diffusivity (kinematic viscosity) to thermal diffusivity. In this Brief Report Pr values are close to unity. The last one is the Biot number, $\mathrm{Bi}=h d / \kappa$, which accounts for heat transfer between the fluid and the atmosphere. Values inside the range [0.8-1.4] are explored here.

As soon as a temperature gradient is imposed, the fluid evolves until an axisymmetric stationary convective motion is reached, called the basic state. This solution is computed similarly to [5,11]. First a Newton-like iterative method is used to solve the problem as the limit of a sequence of linear partial 


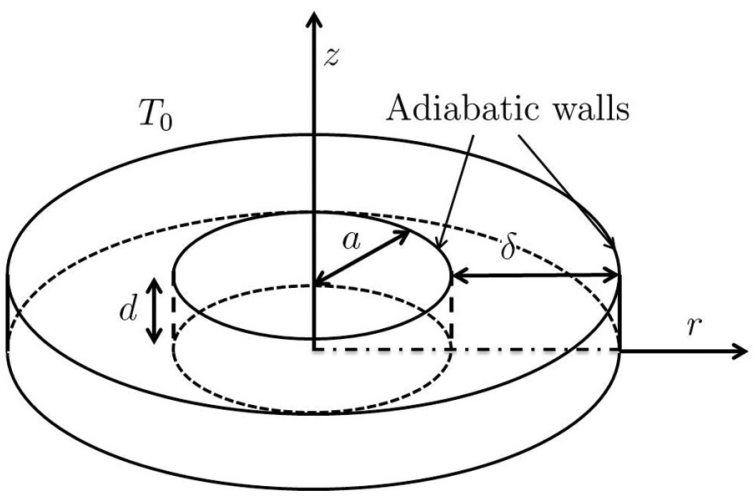

FIG. 1. Sketch of the geometry. Lateral walls are considered adiabatic. The fluid is heated from below and the top surface is open to the atmosphere.

differential equations. The solution is obtained by means of a collocation method [12]. The velocities, temperature, and pressure are approximated by

$$
X(r, z)=\sum_{n=0}^{N} \sum_{m=0}^{M} c_{n m} T^{n}(r) T^{m}(z),
$$

where $T^{N}(x)$ is the Chebyshev polynomial of the first kind of degree $N$. The expansion (4) is substituted into (1), (2), (3), and $\mathrm{BC}(\mathrm{I})$. The resultant equations are then evaluated in the Gauss-Lobatto points, $x_{j}=\cos (\pi j / N), j=0, \ldots, N$. Gauss-Lobatto points are not equispaced but concentrated near the boundaries. This is of special interest when dealing with boundary effects [13]. In order to obtain boundary conditions for the pressure, the momentum equations are projected by the normal of the boundaries and evaluated there. As the pressure is determined up to an additive constant, an arbitrary value for it is fixed in a boundary point.

This method was experimentally validated in [8] for $\operatorname{Pr}=\infty$ and has been recently modified [11] to include Prandtl numbers close to unity. Keeping fixed Biot and Prandtl numbers and increasing $\mathrm{Ra}$ (and thus $\mathrm{Ma}$ ), the basic state becomes unstable and several different bifurcations arise.
TABLE I. Boundary conditions.

\begin{tabular}{lcc}
\hline \hline$z=0$ & $z=d$ & $r=a, a+\delta$ \\
\hline$u_{r}=0$ & $\partial_{z} u_{r}+\mathrm{Ma}_{r} \Theta=0$ & $u_{r}=0$ \\
$u_{\phi}=0$ & $r \partial_{z} u_{\phi}+\mathrm{Ma}_{\phi} \Theta=0$ & $u_{\phi}=0$ \\
$u_{z}=0$ & $u_{z}=0$ & $u_{z}=0$ \\
$\Theta=\Delta T-\left(T_{G} / \delta\right) r$ & $\partial_{z} \Theta+\mathrm{Bi} \Theta=0$ & $\partial_{n} \Theta=0$ \\
\hline \hline
\end{tabular}

The linear stability analysis supplies information about the threshold for Rayleigh numbers and the shape of growing instabilities. Fluid magnitudes are expanded as a Fourier series:

$$
X(r, \phi, z, t)=X_{b}(r, z)+X_{p}(r, z) e^{i k \phi+\lambda t},
$$

where $k$ is the wave number.

The eigenvalues and eigenfunctions of this problem are computed substituting the expansion given in Eq. (5) in Eqs. (1)-(3) and BC (I). After linearizing the problem, a generalized eigenvalue problem $A \bar{X}=B \lambda \bar{X}$ is obtained. A convergence study of this method was carried on in $[8,11]$. Relatively small meshes, 28 points in $r$ and 14 in $z$, are needed to obtain the critical Rayleigh number with eight digit precision.

Due to the presence of the boundary conditions, the matrix $B$ is singular. Thus, not all the eigenvalues have to be finite, and Arnoldi-like methods cannot be used to compute the largest eigenvalues. The best way of computing the eigenvalues in fluid mechanics has been thoroughly studied (see [14]). In the current work, a computational technique specifically designed for thermoconvective problems is used. This technique, developed by Navarro et al. [15], transforms those infinite eigenvalues into a known finite value. The largest eigenvalue obtained through this transformation corresponds to the largest finite eigenvalue of the original problem.

Depending on the symmetries of the growing perturbation, several bifurcations may appear. The shape and class of this solution depend only on the Biot and the Prandtl numbers. The four different competing solutions found are shown in Fig. 2: a stationary roll, similar to the ones of the basic state, region SR, wave number 0 [2]; a hydrothermal wave or oblique

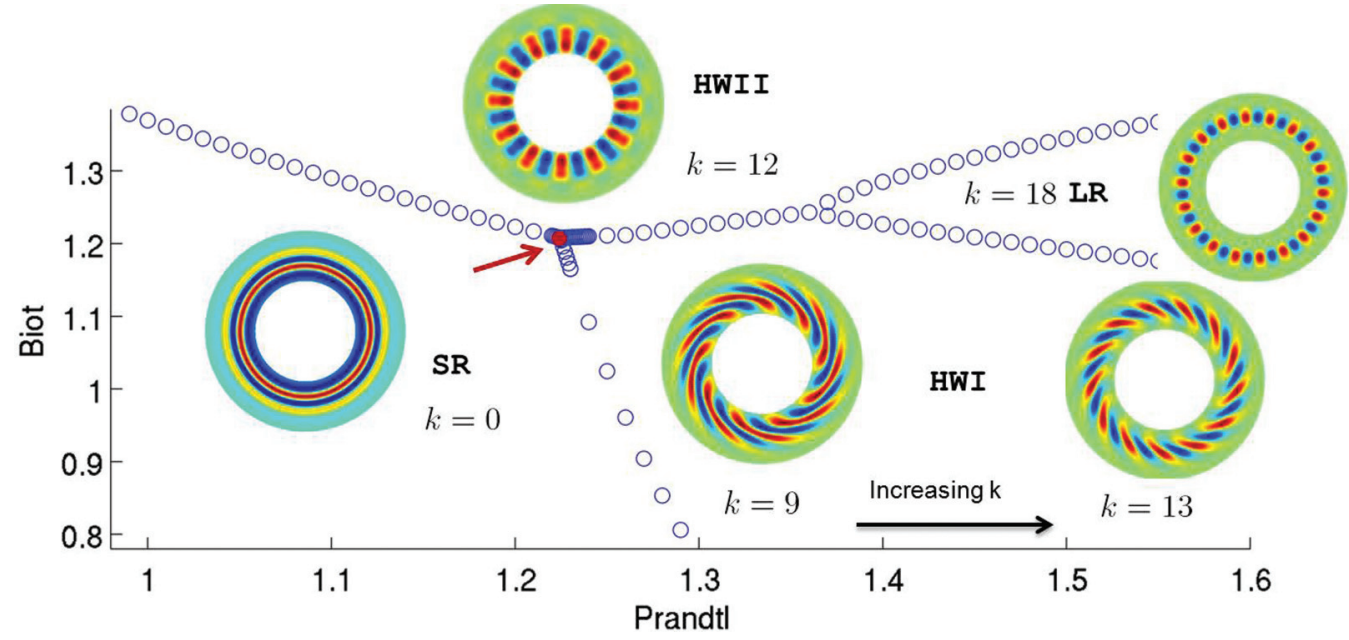

FIG. 2. (Color online) Prandtl-Biot plane stability diagram showing regions I, II, III, and IV. Representative top $r-\phi$ plane isotherms corresponding to aforementioned regions are shown. 


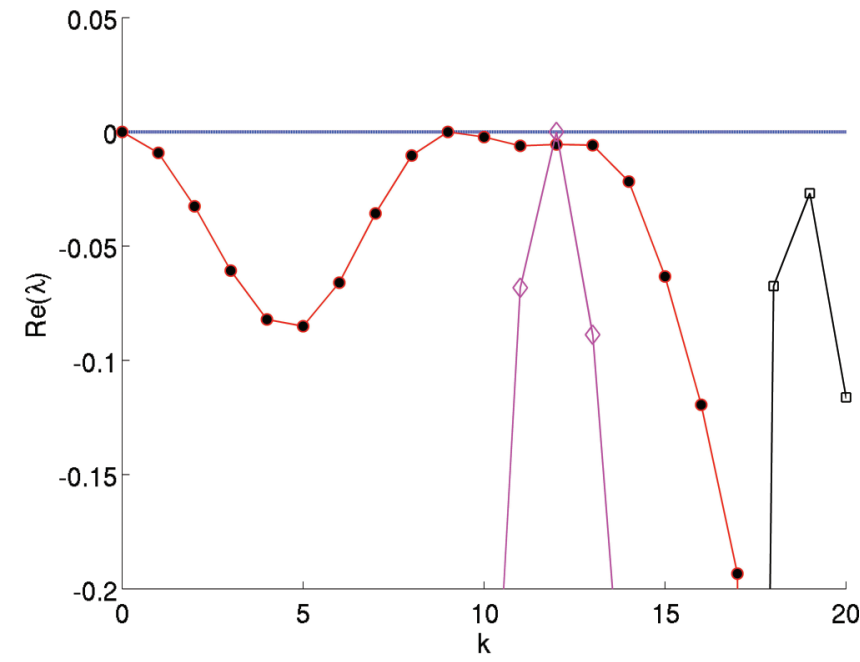

FIG. 3. (Color online) Marginal stability diagram of a codimension-three bifurcation. $\mathrm{Ra}=2258.40, \mathrm{Bi}=1.2082, \mathrm{Pr}=$ 1.2245. Empty points mean a real eigenvalue, whereas bold ones stand for complex eigenvalues. The value $\max \left|\operatorname{Re}\left(\lambda_{0}, \lambda_{9}, \lambda_{12}\right)\right| \leqslant 10^{-5}$. All these curves intersect transversally.

traveling waves, region HWI, wave numbers from 9 to 13 [2]; longitudinal rolls, region LR, wave number 18 [8,16]; and a standing hydrothermal wave of second class or flowerlike wave $[7,17]$, region HWII, wave number 12 . The transition between HWI and HWII was previously reported for $\operatorname{Pr} \sim \infty$, both experimentally and numerically [8], but this is the first time that a transition between SR and HWII can be clearly seen, clarifying Fig. 10(a) of [8]. Moreover, in [8] the transition between HWII and HWI depended only on the aspect ratio and the horizontal temperature gradient, but not on the Biot number as is the case of the current work.

The boundaries of these four regions are made up of codimension-two points, which are the points where the flow becomes unstable for the same critical Rayleigh number for two different wave numbers. Curves separating regions SR, HWI, and HWII intersect at the point marked by an arrow in Fig. 2. Representative top $r-\phi$ plane isotherms have also been plotted in this figure for each of the regions. It is worth mentioning that in previous works we never found the intersection points between these curves (codimension-three points). The marginal stability diagram for this point of the Prandtl-Biot plane is shown in Fig. 3. The points denoted by a shaded circle correspond to complex eigenvalues. At $k=0$ the growing perturbation is a stationary roll (SR), and for $k=9$ the growing perturbation is a hydrothermal wave (HWI). At $k=12$ HWII appears (diamonds). This curve has a very sharp shape since, for $k=10, \operatorname{Re}(\lambda) \simeq-6$. The last curve in this figure corresponds to a solution in the LR region, and thus the real part is always negative at the considered conditions. For $k>20$ the real part of the eigenvalues is below -2 .
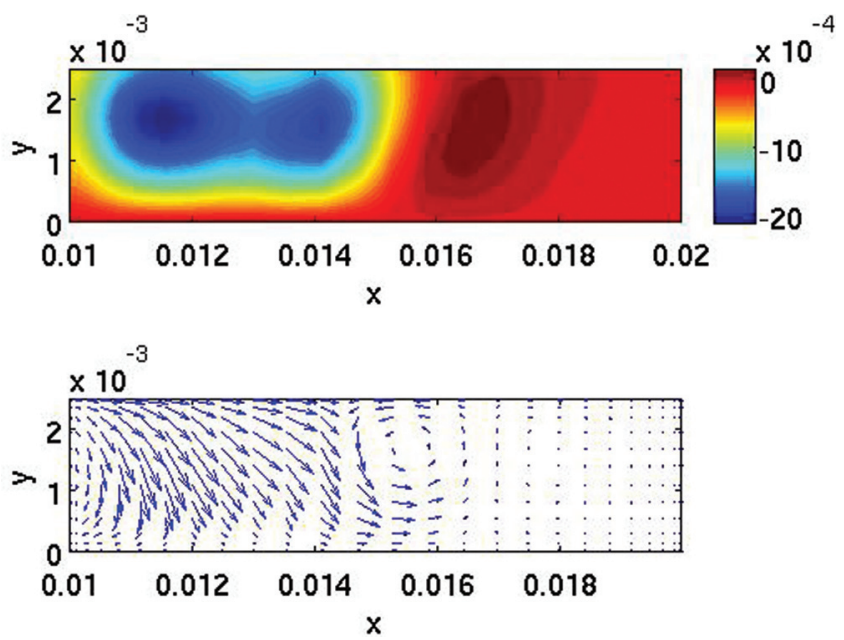

FIG. 4. (Color online) Temperature isotherms and velocity diagram in the $x-z$ plane. Temperature has been normalized dividing it by its maximum.

There exists another codimension-three point at the intersection of HWI, HWII, and LR of Fig. 2. Temperature isotherms and velocity diagrams in the $x-z$ plane of regions SR, HWI, and LR are similar to those shown in [11]. However, the HWII region, found in the current work, shows a very interesting structure as presented in Fig. 4, where a strong roll is present very close to the inner and hotter wall. This structure is similar to the experimental one obtained by Garnier and Chiffaudel [7].

This set of bifurcations could provide a justification of the experimental control of hydrothermal waves reported in [18], where to suppress the hydrothermal waves they use a laser beam that modifies the heat exchange at the surface and thus modifies the Biot number.

In conclusion, a great diversity of transitions has been found in a thermoconvective problem with an imposed constant temperature gradient at the bottom. All the bifurcations are controlled by heat related parameters ( $\mathrm{Ra}, \mathrm{Pr}$, and $\mathrm{Bi}$ ), for a constant Bond number. The domain geometry has been kept constant through all the computations. The four possible patterns for the instabilities have been found in a small region of the Pr-Bi plane. All of them, including the transitions, have been previously reported in experiments, and several features have been recovered in the current results, such as the roll appearing near the hotter side or the multicellular states.

S.H. thanks the Technische Universität Darmstadt for a stay in the Department of Mechanical Engineering, where he finished the code used in this Brief Report. The authors would like to thank Prof. Oberlack, Dr. Khujadze, and Mr. Avsarkisov for their support and kindness. We also are very grateful to the referee for his/her work.
[1] H. Bènard, Rev. Gen. Sci. Pure Appl. 11, 1261 (1900).

[2] M. K. Smith and S. H. Davis, J. Fluid Mech. 132, 119 (1983).

[3] H. Herrero and A. M. Mancho, Phys. Rev. E 57, 7336 (1998).
[4] R. J. Riley and G. P. Neitzel, J. Fluid Mech. 359, 143 (1998).

[5] S. Hoyas, H. Herrero, and A. M. Mancho, J. Phys. A 35, 4067 (2002). 
[6] S. Hoyas, H. Herrero, and A. M. Mancho, Phys. Rev. E 66, 057301 (2002).

[7] N. Garnier and A. Chiffaudel, Eur. Phys. J. B 19, 87 (2001).

[8] S. Hoyas, H. Herrero, A. M. Mancho, N. Garnier, and A. Chiffaudel, Phys. Fluids 1, 054104 (2005).

[9] D. Schwabe, A. Zebib, and B.-C. Sim, J. Fluid Mech. 491, 239 (2003).

[10] S. Chandrasekhar, Hydrodynamic and Hydromagnetic Stability (Dover, New York, 1961).

[11] A. J. Torregrosa, S. Hoyas, M. J Perez-Quiles, and J. M. MompoLaborda, Commun. Comput. Phys. 13, 428 (2013).
[12] S. A. Orszag, Stud. Appl. Math. 51, 253 (1972).

[13] J. Jiménez and S. Hoyas, J. Fluid Mech. 611, 215 (2008).

[14] V. Theofilis, Annual Review of Fluid Mechanics 43, 319 (2011).

[15] M. C. Navarro, H. Herrero, and S. Hoyas, Commun. Comput. Phys. 5, 649 (2009).

[16] J. Burguete, N. Mukolobwiez, F. Daviaud, N. Garnier, and A. Chiffaudel, Phys. Fluids 13, 2773 (2001).

[17] E. Favre, L. Blumenfeld, and F. Daviaud, Phys. Fluids 9, 1473 (1997).

[18] S. Benz, P. Hintz, R. J. Riley, and G. P. Neitzel, J. Fluid Mech. 359, 165 (1998). 